dass die in Fäkalien enthaltenen Anteile von Stickstoff und Phosphor einen knappen Rohstoff darstellen und als Dünger unverzichtbar sind. Hundert Jahre später ist man klüger. Inzwischen ist die chemische Kompost-Toilette mit dem dazugehörigen logistischen System jedermann vertraut. Auch ist die energieintensive Stickstoffgewinnung aus der Luft als energieverschwendende Notlösung erkannt, die nicht mehr lange durchzuhalten ist. Vor diesem Hintergrund erscheint die energieaufwendige Klärung der via Schwemmkanalisation abgeführten und unsinnigerweise auch noch vermischten Abwässer systematisch nicht naheliegend. (205) Jedenfalls ist sie nicht offensichtlich optimal. Sie besteht nämlich in der Vernichtung der organischen Anteile und Nährstoffe sowie in der "Eliminierung“ von Stickstoff und Phosphor samt deren anschließender Deponierung. Doch ein Großsystem kann man nicht schlagartig, auf einmal ändern. Die Kunst besteht darin, Pfade von einem suboptimalen Zustand zu einem deutlich besseren zu finden. Die Aufgabe erinnert an die, die beim Rentensystem ansteht, welches man in ein System der Kapitaldeckung überführen will.

Formal handelt es sich um das lobenswerte Unikum einer Habilitationsschrift (an der Universität Kassel) eines Wissenschaftlers, der seine Kompetenz in vielfältiger Beratungstätigkeit in wasserwirtschaftlichen Zusammenhängen erworben hat. Bei aller sympathischen historischen und begriffsgeschichtlichen Vertiefung, also akademischen Leckerbissen, - der Autor weiß auch praktisch und im aktuellen Detail sehr genau, wovon er redet. Die Fokussierung auf das Hessische Ried ist im übrigen nicht Ausdruck von Kleinkrämerei, sondern Programm. Es geht dem Autor um die Option einer durchgängigen Regionalisierung, einer Stärkung der örtlichen Ressourcen und Ausgleichfähigkeiten gegen die Tendenzen einer zunehmenden Einbindung in eine vernetzte überregionale Versorgung, welche die Krankheitssymptome einer Region zugleich lindert und exportiert. Und die damit die Bedingungen für eine Krisenentwicklung, in die eine wachsende Zahl von Beteiligten einbezogen würde, allererst schafft.

\section{Bibliographische Angaben}

Kluge, Th., 2000: Wasser und Gesellschaft. Von der hydraulischen Maschinerie zur nachhaltigen Entwicklung - ein Fallbeispiel. Opladen: Leske + Budrich, 254 S. DM 48,--. (Reihe Soziologie und Ökologie; Bd. 3).

\section{MICHAEL LATZER (Hrsg.): Mediamatik- politik für die Digitale Ökonomie: eCommerce, Qualifikation und Markt- macht in der Informationsgesellschaft. Innsbruck, Wien, München: Studien- Verlag, 2000. 362 S. DM 61,50. ISBN 3- 7065-1518-0 (Beiträge zur Medien- und Kommunikationsgesellschaft, Bd. 6, hrsg. v. der Österreichischen Gesell- schaft für Kommunikationsfragen - ÖGK)}

\section{Rezension von Carsten Orwat, ITAS}

Der vorliegende Band enthält Texte, die vom Projektteam der Forschungsstelle für institutionellen Wandel und europäische Integration (http://www.iwe.oeaw.ac.at) der Österreichischen Akademie der Wissenschaften im Auftrag des damaligen österreichischen Bundesministeriums für Wissenschaft und Verkehr erstellt wurden. Im Mittelpunkt der Studie stehen die sogenannte „Mediamatik“ und die „,digitale Ökonomie“. Beide werden in ihren besonderen (ökonomischen) Charakteristika analysiert, um darauf aufbauend ihre Auswirkungen für die Politik und den resultierenden Reformbedarf abzuleiten. Die Studie hat den Anspruch, als inhaltliche Grundlage für politische Maßnahmen zu dienen.

Der synthetische Begriff „Mediamatik“ (Mediamatik $=$ elektronische Medien + Telematik; Telematik = Telekommumikation + Informatik) soll die technische, inhaltliche und wirtschaftliche Konvergenz von Telekommunikation, Computer (Hardware und Software) und Rundfunk (Medien) verdeutlichen. Die Autoren sind bestrebt, mit dem „Mediamatik“Begriff mehr abzudecken als den Bereich der Informations- und Kommunikationstechnologien (IKT), nämlich insbesondere auch den 
Bereich der Inhaltsproduktion. Die Kernaussage des Buches lässt sich so zusammenfassen: Die Konvergenz der technischen Medien erfordere auch eine Integration der politischen Institutionen und Funktionen im Sinne einer verbesserten Abstimmung von Zuständigkeiten und gesetzlichen Normen. Um Effizienzverluste eines separaten und unkoordinierten staatlichen Handels in den Bereichen Telekommunikation, Computer und Rundfunk zu vermeiden, fordern die Autoren eine sogenannte ,integrierte Mediamatikpolitik“, die mittels institutioneller Neuerungen, geeigneter Rahmenbedingungen und staatlicher Eingriffe den Übergang zur ,digitalen Ökonomie “ fördere. Unter einer „digitalen Ökonomie“ (synonym auch Netz- bzw. Internet-Ökonomie) wird in der Studie das Ergebnis eines Transformationsprozesses des Großteils der Wirtschaft verstanden (S. 41ff.). In ihr würden Netzwerkeffekte, wachsende Skalenerträge und positive Rückkopplungseffekte eine dominierende Rolle spielen. Sie könnten „Marktversagen" hervorrufen und einen politischen Handlungsbedarf begründen. Zur ,,digitalen Ökonomie" wird auch der elektronischen Handel gezählt, der in erster Linie als Handel zwischen verschiedenen Unternehmen oder zwischen Unternehmen und Konsumenten verstanden wird, wobei einige oder alle Handelstransaktionen über das Internet abgewickelt werden (S. 62-68). Die Studie richtet ihren Betrachtungsschwerpunkt auf den elektronischen Handel zwischen Unternehmen und Konsumenten („,business-to-consumer E-Commerce“ oder vereinfacht B2C E-Commerce). Nach dieser knappen Übersicht über die im Buch verwendeten Begriffe und Abgrenzungen soll im Folgenden auf die einzelnen Kapitel eingegangen werden.

Im Kapitel 1 (,Die europäische Informationsgesellschaft als politisch-ökonomische Herausforderung") stellt Michael Latzer die Initiativen der Europäischen Union auf dem Gebiet der „Informationsgesellschaft“ vor. Die genannten Programme zielten in erster Linie auf die Liberalisierung der Telekommunikationsmärkte und des Fernsehsektors ab und sollten einen Rechtsrahmen für den elektronischen Geschäftsverkehr schaffen. Daneben wird in dem Kapitel das methodische Vorgehen der Analysen in den nachfolgenden Kapiteln vor- gestellt. In erster Linie seien Ansätze der Industrieökonomie und der Neuen Politischen Ökonomie angewandt worden.

Latzer und Stefan W. Schmitz beleuchten im Kapitel 2 (,,Mediamatik und Digitale Ökonomie als zentrale Kennzeichen der Informationsgesellschaft") detailliert die besonderen Eigenschaften der „Mediamatik“, der „Mediamatik“-Politik und der „digitalen Ökonomie“. Mit Beispielen veranschaulichen die Autoren, dass die nach technischen Kriterien getrennt in Wissenschaft und Politik erfolgte Behandlung der Bereiche Medien (Rundfunk) und Telekommunikation obsolet sei. Zum Beispiel sei es in verschiedenen europäischen Rechtssystemen unklar, ob die Verbreitung von Nachrichten, Audio-Inhalten, Videos usw. über das Internet nun den Regulierungen aus dem Rundfunk-, Presse- oder Telekommunikationsbereich unterstehe (S. 35f.). Mit der MediamatikPolitik solle Abhilfe geschaffen werden. Sie behandele die Telekommunikation, Medien und Informationstechnologien integrativ in einer umfassenden und geschlossenen Regulierung der elektronischen Kommunikation. In ihrem Gefolge bedürfe es institutioneller Neuerungen sowie einer verbesserten Verteilung der politischen Zuständigkeiten und Aufgabenbereiche.

Im Kapitel 3 („Aufgabenbereiche für die Politik") finden sich die Beiträge, in denen das geforderte staatliche Handeln konkretisiert wird. Dazu werden zunächst von Schmitz im Kapitel 3.1 („Die Förderung des Business-toConsumer eCommerce") die Bausteine einer ECommerce-Politik ausführlich dargestellt. Im Einzelnen beziehen sie sich auf die Vertrauensbildung, den Verbraucherschutz, den Datenschutz, die Zoll- und Steuerpolitik, die Förderung von Klein- und Mittleren Unternehmen sowie auf das elektronische Geld. Dabei werden unterschiedliche Politikkonzepte, wie z. B. die staatliche Regulierung oder eine marktnahe Selbstregulierung, argumentativ abgewogen. Ebenso werden bereits durchgeführte regulative Maßnahmen auf internationaler, supranationaler bzw. europäischer und österreichischer Ebene beschrieben. In einem Unterabschnitt des Kapitels stellt Peter Slominski die rechtlichen Rahmenbedingungen $\mathrm{zu}$ elektronischen Signaturen dar. Im anschließenden Kapitel 3.2 behandelt Martin Wörter die ,Qualifikations- 
anforderungen im Mediamatiksektor", wobei das Mediamatik-relevante Ausbildungsniveau, die staatlichen und betrieblichen Ausbildungsmaßnahmen und der weitere Bedarf insbesondere für Österreich erläutert werden.

Anschließend widmet sich Natascha Just der Wettbewerbspolitik als einem weiteren Bestandteil der Mediamatik-Politik (Kapitel 3.3, „Die Kontrolle der Marktmacht in der Mediamatik"). An dieser Stelle wird noch einmal eindrücklich die Problematik sichtbar, dass im Zuge der Konvergenz zwei Regulierungskonzeptionen aufeinander treffen würden: Zum einen sei der Telekommunikations- und Mediensektor mit spezifischen und detaillierten Regulierungen überzogen, und zum anderen unterstehe der Sektor der Informations- und Kommunikationstechnologie lediglich den Rahmenbedingungen des Wettbewerbsrechts. Just greift sich zur genaueren Betrachtung die Marktmachtkontrolle heraus und beschreibt daran die gegenwärtigen Herausforderungen an die Wettbewerbspolitik. Hierzu gehöre beispielsweise, dass unterschiedliche und damit diskriminierende Regulierungen, die für Betreiber entweder von Kabelfernsehen oder von Telekommunikationsnetzen gelten, durch technik-neutrale Regulierungen ersetzt werden sollen. Zudem sei zur wettbewerbsrechtlichen Beurteilung von Unternehmenszusammenschlüssen oder marktbeherrschendem Verhalten eine klare Definition des relevanten Marktes unerlässlich. Eine derartige Definition sei jedoch insbesondere für die Bereiche Telekommunikation, digitales Fernsehen und Internet problematisch (S. 267-275).

Latzer und Schmitz liefern im Kapitel 4 (,Business-to-Consumer eCommerce in Österreich: eine empirische Untersuchung") die Ergebnisse einer empirischen Untersuchung zur Bedeutung und zu den Eigenschaften der drei Phasen des B2C E-Commerce. Es werden die Informationsphase, die Vereinbarungsphase (Vertragsverhandlung und -abschluss) und die Abwicklungsphase (Bezahlung, Lieferung) unterschieden. 1.000 regelmäßige InternetNutzer in Österreich wurden mittels Fragebögen im Internet befragt. Ziel der Untersuchung war es, Erkenntnisse über die Struktur (einschließlich Zahlungsgewohnheiten, Volumen der Nachfrage und Umsatzsteuer) und über die Motive sowie Entwicklungshemmnisse des
B2C E-Commerce zu gewinnen. Bei den Ergebnissen sei hier nur auf einige Entwicklungshemmnisse verwiesen, wie die Unklarheiten hinsichtlich des Verbraucher- und Datenschutzes, die fehlende Möglichkeit, das Produkt vor dem Kauf zu inspizieren, und die von den Internet-Nutzern als unsicher und kompliziert eingeschätzte Bezahlung im Internet.

Im abschließenden Kapitel 5 (,Transformation der Staatlichkeit - Schlussfolgerungen für die Politik") fasst Latzer die Erkenntnisse der vorhergehenden Kapitel zusammen und verdeutlicht auf einer übergeordneten Ebene die Vorstellungen zu einer neuen Staatlichkeit in der ,digitalen Ökonomie“. Hierbei handele es sich nicht bloß um eine punktuelle Adaption neuer Politikmuster, sondern um eine umfassende Transformation der Staatlichkeit. Zur Staatlichkeit zählt Latzer unter anderem die institutionelle Ordnung, die Verteilung von Aufgaben, Zuständigkeiten und Befugnissen, die inhaltliche Ausrichtung der Politik, die verwendeten Instrumente, das Netzwerk der Akteure sowie die organisatorischen Spezifika im Mediamatik-Sektor. Hierbei formuliert und diskutiert er Thesen zur Transformation der Staatlichkeit, die zum Teil den Charakter von Forderungen haben, zum Teil tatsächliche Entwicklungen beschreiben. Es sei hier beispielhaft hervorgehoben, dass die Verlagerung politischer Zuständigkeiten von nationaler auf supranationale oder internationale Ebene erfolge, dass zunehmend weite und auslegungsbedürftige Begriffe zusammen mit einer verbreiteten Kooperation von Regulierern und Regulierten anstelle von detaillierten staatlichen Regulierungen angewendet werde sowie dass staatliche Regulierungen zunehmend durch marktnahe Selbstregulierung ersetzt würden. An dieser Stelle wäre jedoch eine eingehendere Analyse der Vor- und Nachteile derartiger Tendenzen der Transformation von Politik für den Leser von Wert gewesen.

\section{Fazit}

Das vorliegende Buch liefert eine einführende Übersicht zu dem höchst aktuellen, jedoch komplexen und diffizilen Themenbereich der Regulierungen in den konvergierenden Bereichen Telekommunikation, Informationstechnik 
und Medien, verdeutlicht insbesondere anhand von Fragen der Regulierung des Internet und des elektronischen Handels. Zu den Verdiensten der Studie kann gezählt werden, dass das Themenfeld nicht nur umfassend und detailliert beschrieben, sondern auch thematisch gut strukturiert wurde. Neben den Skizzen und Analysen der existierenden Regulierungskonzepte sind vor allem die zahlreichen Vorschläge und Denkanstöße, wie sie in Zukunft gestaltet sein sollten, von Nutzen. Seinen Übersichtscharakter erhält das Buch dadurch, dass analytische Diskussionen, wie z.B. über die genannten ökonomischen Charakteristika des Mediamatik-Bereichs (Netzwerkeffekte, steigende Skalenerträge etc.), sich nicht im Detail verlieren. Sie werden vielmehr nur in soweit geführt, wie es für die nachfolgenden Erörterungen der Konzepte einer Mediamatik-Politik von Nöten ist. Dementsprechend sind die Analysen allerdings auch nicht erschöpfend, und so stellt sich zum Beispiel die Frage, ob die beschriebenen Charakteristika eindeutig zu den genannten Marktstrukturen führen oder ob nicht auch gegenläufige Tendenzen existieren. Den positiven Netzwerkeffekten, d. h. dem höheren Nutzen des einzelnen Teilnehmers bei Ausweitung des Teilnehmerzahl, und der Tendenz zu Monopolen können auch Nachteile einer größeren Netznutzung entgegenstehen, wie z. B. die Überfüllung der Netzwerke, die höheren Kosten ihrer Organisation oder dadurch, dass mit der einheitlichen Netzwerkleistung unterschiedliche Präferenzen nicht befriedigt werden. Verschiedenste technische und ökonomische Gegebenheiten in den vielfältigen Bereichen der „digitalen Ökonomie“ erlauben demnach keine allgemeinen Schlussfolgerungen hinsichtlich Marktstrukturen, abgeleitetem „Marktversagen“ und Regulierungsnotwendigkeiten. An diesen Stellen wird leicht weiterer Forschungsbedarf deutlich, mit dem differenziertere Aussagen getroffen werden könnten.

\section{BÜCHER KURZ VORGESTELLT}

\author{
ORIO GIARINI, WALTER R. STAHEL: Die \\ Performance-Gesellschaft: Chancen und \\ Risiken beim Übergang zur Service Econo- \\ my. Marburg: Metropolis Verlag, 2000. \\ 320 S. DM 39,80. ISBN 3-89518-320-2
}

Dieses Buch handelt von Wirtschaft und Arbeit; es analysiert die Entwicklung der letzten zweihundert Jahre (die Industriegesellschaft) und zeigt mögliche Zukünfte (die Dienstleistungsgesellschaft), welche der Gesellschaft große neue Chancen bieten, aber auch einen Umbau der Wirtschafts- und Arbeitswelt bedingen, der wieder neue Möglichkeiten, aber auch neue Risiken mit sich bringt.

Zuerst in englischer Sprache erschienen, seither in mehrere Sprachen übersetzt, ist dieser Text in Japan in kurzer Zeit zu einem Referenzwerk für ein nachhaltiges Wirtschaften geworden.

Die Autoren haben ihre langjährige Erfahrung in strategischem Denken, welches sie in Industrie, Forschung und Lehre entwickelt und erfolgreich angewandt haben, in diesem Buch zusammengefasst. Die Chancen der Zukunft liegen in einem nachhaltigen Wirtschaften, welches auf drei Pfeilern aufbaut:

- einem systemischen Denken und einer Optimierung auf Systemebene, welche die heutige Produktoptimierung ablösen werden,

- $\quad$ einem Einbezug des Faktors „Zeit“ in die wirtschaftliche Optimierung, welcher wegführt von der traditionellen Betrachtung der Wirtschaft als Fluss zu einer Betrachtung der Wirtschaft als See,

- einem neuen zentralen Wertbezug der Wirtschaft: der Nutzungswert wird zunehmend den Tauschwert ersetzen, und der Verkauf von Leistung über eine Zeitperiode wird den einmaligen Verkauf von Gütern ablösen. 